\title{
Intervenciones psicosociales en atención primaria: evidencia publicada y relato de la experiencia del Servicio de Medicina Familiar y Comunitaria en Argentina
}

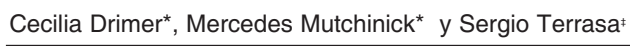

\begin{abstract}
Resumen
En una primera parte, este artículo describe las características distintivas de la medicina familiar y los principales resultados de las intervenciones psicosociales realizadas por médicos generales cuando fueron comparadas contra un grupo control, de acuerdo a la revisión sistemática publicada por Huibers y col. Una segunda parte este artículo comunica las principales características de un dispositivo de abordaje psicosocial denominado FOCO (Familia, Orientacion y COntexto) desarrollado y actualmente aplicado por los médicos de familia del Servicio de Medicina Familiar y Comunitaria de Hospital Italiano de Buenos Aires; así como la ficha de registro que procura orientar al profesional actuante para conducir un proceso de atención ordenado y con objetivos precisos, y a registrarlo en la historia clínica.
\end{abstract}

\section{Abstract}

In the first part, this article describes the hallmarks of family medicine practice and the main results of psychosocial interventions made by general practitioners when these were compared against a control group, according to a systematic review published by Huibers et al. A second part of this paper reports the main characteristics of a psychosocial approach device named FOCO (family, counseling and context) currently developed and applied by family physicians from the Family and Community Medicine Department at Hospital Italiano de Buenos Aires, as well as the registration form that helps physicians to follow an structured interview.

Palabras clave: intervención psicosocial, medicina familiar, historia clínica.Key words: psychosocial intervention, family medicine, health record.

Drimer C, Mutchinick M y Terrasa S. Intervenciones psicosociales en atención primaria: evidencia publicada y relato de la experiencia del Servicio de Medicina Familiar y Comunitaria en Argentina. Evid Act Práct Ambul. Jul-Set 2012;15(3):100-103

\begin{abstract}
Características distintivas del abordaje en medicina familiar La Medicina Familiar se convirtió en una especialidad certificada en la década de 1960, definida como una especialidad horizontal que tiene como una de las aristas de su paradigma comprender y reubicar el problema orgánico y/o emocional de los pacientes en el contexto de su situación vital y dentro de un marco familiar, para lo cual integra las ciencias biológicas, las clínicas y las de la conducta1. De esta manera, la evaluación del paciente desde una concepción bio-psico-social ${ }^{2 t \dagger}$, teniendo en cuenta sus distintas emociones, sus vínculos, su familia y su contexto, contribuye enormemente al trabajo cotidiano del médico de familia, que da importancia tanto a los aspectos objetivos de una enfermedad, como a los subjetivos ${ }^{\ddagger \neq}$ (p. ej. las relaciones, las emociones y la autorreflexión) ${ }^{1,3}$.
\end{abstract}

A través de este paradigma, se intenta ofrecer un espacio específico para el armado de una estrategia personalizada que genere un cambio favorable en la salud. Su principal virtud es que provee información valiosa para conocer al paciente como persona, de manera tal que el diagnóstico del problema se realice en múltiples niveles y la estrategia terapéutica pueda ser más eficaz. Más allá de que la atención cotidiana del médico de familia incluye el paradigma bio-psico-social antes descripto, este puede en algunas ocasiones ofrecer a sus pacientes $\mathrm{y} / \mathrm{o}$ famlias dispositivos de "atención psicosocial ampliada", que en la blibliografía suelen ser mencionados como intervenciones psicosociales en atención primaria. En la tabla 1 resumimos los principales resultados de algunas de ellas cuando fueron comparadas con algún grupo control, como fuera comunicada por Huibers $\mathrm{M} \mathrm{y} \mathrm{col}^{4}$, en su revisión sistemática Cochrane.

Tabla 1: resultados principales de la revisión sistemática publicada por Huibers M y col. (2008).

\begin{tabular}{|c|c|c|c|}
\hline Trabajo & Participantes & Intervención y comparación & Resultados \\
\hline $\begin{array}{l}\text { Blankenstein } \\
2001\end{array}$ & $\begin{array}{l}162 \text { pacientes somatizadores ( } 20 \text { a } 45 \text { años) } \\
\text { con más de } 15 \text { visitas durante tres años, o más } \\
\text { de cinco síntomas de somatización. Diez } \\
\text { médicos generalistas. } \\
\text { No informa sobre la aleatorización de los } \\
\text { pacientes. }\end{array}$ & $\begin{array}{l}\text { Intervención ( } n=75) \text { : dos a tres sesiones de } \\
\text { diez a } 30 \text { minutos con la estrategia de } \\
\text { reatribución (trabajo con la preocupación por la } \\
\text { enfermedad y la comprensión de los } \\
\text { sentimientos) luego de un programa de } 20 \mathrm{~h} \text { de } \\
\text { entrenamiento para los profesionales. } \\
\text { Control ( } n=87) \text { : atención habitual. }\end{array}$ & $\begin{array}{l}\text { Los asignados a la rama intervención tuvieron } \\
\text { mejores puntuaciones en todas las medidas de } \\
\text { resultado (consumo de recursos médicos, salud } \\
\text { subjetiva y licencia por enfermedad). } \\
\text { Ninguno se recuperó totalmente. }\end{array}$ \\
\hline $\begin{array}{l}\text { Huibers } \\
2004\end{array}$ & $\begin{array}{l}151 \text { pacientes de un servicio de salud } \\
\text { ocupacional con fatiga severa y ausentismo } \\
\text { laboral ( } 18 \text { a } 65 \text { años). Nueve médicos } \\
\text { generalistas. No informa sobre la aleatorización } \\
\text { de los pacientes. }\end{array}$ & $\begin{array}{l}\text { Intervención ( } n=76) \text { : cinco a siete sesiones de } \\
\text { terapia cognitivo-conductual de } 30 \text { minutos de } \\
\text { duración durante cuatro meses con } \\
\text { entrenamiento previo de los profesionales y } \\
\text { supervisión durante el proceso. } \\
\text { Control }(n=75) \text { : abordaje usual. }\end{array}$ & $\begin{array}{l}\text { No se constataron diferencias significativas a los } \\
\text { cuatro, ocho y } 12 \text { meses. }\end{array}$ \\
\hline $\begin{array}{l}\text { Larisch } \\
2004\end{array}$ & $\begin{array}{l}127 \text { pacientes somatizadores } \\
\text { (18 a } 65 \text { años). La aleatorización fue realizada } \\
\text { por profesional ( } 20 \text { en el grupo intervención y } \\
17 \text { en el control). }\end{array}$ & $\begin{array}{l}\text { Intervención ( } n=73) \text { : atención psicosocial } \\
\text { habitual de APS más intervención de } \\
\text { reatribución. } \\
\text { Control ( } n=75) \text { : atención psicosocial habitual. } \\
\text { Los médicos fueron entrenados durante } 80 \\
\text { horas y ambos grupos recibieron sesiones de } 6 \\
\text { a } 20 \text { minutos de duración durante seis meses. } \\
\text { En el grupo tratamiento los médicos recibieron } \\
\text { 12h de entrenamiento adicional. }\end{array}$ & $\begin{array}{l}\text { Sin diferencias significativas a los } 3 \text { y } 6 \text { meses en } \\
\text { cuanto a síntomas físicos, ansiedad y depresión, } \\
\text { calidad de vida o salud mental, salvo en el caso } \\
\text { de los síntomas físicos a los } 6 \text { meses. } \\
\text { Sin descripción adecuada de los resultados a los } \\
12 \text { meses. }\end{array}$ \\
\hline
\end{tabular}

* Servicio de Medicina Familiar y Comunitaria del Hospital Italiano de Buenos Aires. mercedes.mutchinick@ hospitalitaliano.org.ar * ¿ Departamento de Salud Pública del Instituto Universitario Hospital Italiano de Buenos Aires.

"El modelo biomédico tradicional emplea la estrategia de descartar causas orgánicas antes de explorar otras dimensiones, lo que lleva implícito el concepto de que la enfermedad orgánica posee una importancia primaria y que los fenómenos físicos y mentales tienen fronteras muy delimitadas. Sin embargo, ya desde 1946 la OMS adoptó en su constitución la definición: «La salud es un estado de completo bienestar físico, mental y social, y no solamente la ausencia de afecciones o enfermedades.”

\#La experiencia del paciente siempre es única y subjetiva, siendo la tarea del profesional de la salud comprender su contexto, teniendo en cuenta también a las emociones, como reflejo de representaciones físicas y mentales que están modeladas por pautas biológicas, históricas, familiares y culturales. 


\begin{tabular}{|c|c|c|c|}
\hline Lidbeck 1997 & $\begin{array}{l}50 \text { pacientes y } \\
\text { (30 a } 60 \text { años) que cumplian criterios de } \\
\text { trastornos funcionales específicos o síntomas } \\
\text { somáticos inexplicables, reclutados por } \\
\text { médicos generalistas en una clínica } \\
\text { ambulatoria. } \\
\text { La aleatorización estuvo sesgada por la } \\
\text { diferencia de pacientes en ambos grupos. }\end{array}$ & $\begin{array}{l}\text { Intervención ( } n=33) \text { : grupo de terapia } \\
\text { cognitivo-conductual, con ocho sesiones de } \\
\text { tres horas durante dos meses. } \\
\text { Control ( } n=17) \text { : lista de espera. } \\
\text { Programa de entrenamiento para profesionales, } \\
\text { sin referencia a la supervisión. }\end{array}$ & $\begin{array}{l}\text { Después de seis meses de seguimiento el grupo } \\
\text { asignado a la intervención tuvo resultados } \\
\text { superiores a los del grupo control en términos de } \\
\text { su conducta frente a la enfermedad y el uso de } \\
\text { medicación, sin diferencias significativas en su } \\
\text { ansiedad, depresión, sueño y en sus problemas } \\
\text { sociales. }\end{array}$ \\
\hline McIntosh 1997 & $\begin{array}{l}159 \text { pacientes mayores } 15 \text { años con alta tasa } \\
\text { de consumo de alcohol (CAGE } 1 \text { item o más, } 0 \\
\text { consumo de cuatro o más tragos diarios) } \\
\text { reclutados por enfermeras en un centro de } \\
\text { medicina familiar. } \\
\text { No se informó la forma de la aleatorización, ni } \\
\text { el mecanismo de supervisión y/o } \\
\text { entrenamientos de dichos profesionales. }\end{array}$ & $\begin{array}{l}\text { Intervención } 1 \text { ( } \mathrm{n}=40) \text { : intervención } \\
\text { cognitivo-conductual por un médico generalista } \\
\text { (dos sesiones de } 30 \text { minutos durante } \\
\text { semanas). } \\
\text { Intervención } 2 \text { ( } \mathrm{n}=66 \text { ): intervención } \\
\text { cognitivo-conductual por un enfermero, dos } \\
\text { sesiones de } 30 \text { minutos durante dos semanas. } \\
\text { Control ( } \mathrm{n}=53 \text { ): consejo breve brindado por el } \\
\text { médico generalista durante una sesión de } \\
\text { cinco minutos. }\end{array}$ & $\begin{array}{l}\text { Sin diferencias significativas entre ambos } \\
\text { después de } 12 \text { meses de seguimiento. }\end{array}$ \\
\hline $\begin{array}{l}\text { Mynors-Wallis } \\
1995\end{array}$ & $\begin{array}{l}91 \text { pacientes de } 18 \text { a } 65 \text { años de edad con } \\
\text { depresión mayor (13 o más puntos en la escala } \\
\text { de Hamilton 13) reclutados por médicos } \\
\text { generalistas. } \\
\text { Participaron dos médicos generalista y un } \\
\text { psiquiatra. } \\
\text { La aleatorización fue estratificada para } \\
\text { asegurar que los tres grupos tuvieran } \\
\text { pacientes con trastornos de depresivos de } \\
\text { severidad equivalente. }\end{array}$ & $\begin{array}{l}\text { Intervención } 1 \text { ( } \mathrm{n}=30) \text { : tratamiento de } \\
\text { resolución de problemas. } \\
\text { Intervención } 2 \text { ( } \mathrm{n}=31): 50 \text { a } 150 \mathrm{mg} \text { de } \\
\text { amitriptilina y cuidados habituales. } \\
\text { Control ( } \mathrm{n}=30) \text { : placebo y atención habitual. } \\
\text { Todos los grupos recibieron seis sesiones de } \\
30 \text { a } 60 \text { minutos durante } 12 \text { semanas. } \\
\text { Los médicos recibieron entrenamiento, sin } \\
\text { reportarse si hubo supervisión. }\end{array}$ & $\begin{array}{l}\text { Luego de } 12 \text { semanas de seguimiento los } \\
\text { resultados (puntajes de Beck y Hamilton para } \\
\text { depresión y para puntaje de desempeño social) } \\
\text { del grupo "Intervención 1" fueron superiores a los } \\
\text { del grupo control. No se observaron diferencias } \\
\text { significativas en los síntomas psicológicos. No } \\
\text { hubo diferencias significativas en los resultados } \\
\text { entre los grupos a ambas intervenciones. }\end{array}$ \\
\hline $\begin{array}{l}\text { Mynors-Wallis } \\
2000\end{array}$ & $\begin{array}{l}151 \text { pacientes de } 18 \text { a } 65 \text { años con depresión } \\
\text { mayor reclutados por } 24 \text { médicos generalistas. } \\
\text { Participaron tres médicos generalistas y dos } \\
\text { enfermeros. } \\
\text { La aleatorización fue estratificada para } \\
\text { asegurar que los tres grupos tuvieran } \\
\text { pacientes con trastornos de depresivos de } \\
\text { severidad equivalente. }\end{array}$ & $\begin{array}{l}\text { Intervención } 1 \text { ( } \mathrm{n}=39 \text { ): tratamiento de } \\
\text { resolución de problemas por médicos } \\
\text { generalistas } \\
\text { Intervención } 2 \text { ( } \mathrm{n}=41 \text { ): tratamiento de } \\
\text { resolución de problemas por enfermeros. } \\
\text { Intervención } 3 \text { ( } \mathrm{n}=36) \text { : } 100 \text { mg de fluvoxamina } \\
\text { ó } 20 \text { mg de paroxetina y cuidados habituales. } \\
\text { Intervención } 4 \text { ( } \mathrm{n}=35 \text { ): combinación de } \\
\text { tratamientos (uso de medicación por parte del } \\
\text { médico y tratamiento de resolución de } \\
\text { problemas por el enfermero) } \\
\text { A todos los grupos se les ofreció seis sesiones } \\
\text { de } 30 \text { a } 60 \text { minutos durante } 12 \text { semanas (salvo } \\
\text { el grupo cuatro, a quienes se les ofreció } 12 \text { ). } \\
\text { Hubo un programa de entrenamiento y } \\
\text { supervisión para todos los profesionales. }\end{array}$ & $\begin{array}{l}\text { Después de } 52 \text { semanas de seguimiento: sin } \\
\text { diferencias significativas en los grupos de } \\
\text { depresión, síntomas psicológicos o desempeño } \\
\text { social. }\end{array}$ \\
\hline Richmond 1985 & $\begin{array}{l}200 \text { tabaquistas de } 16 \text { a } 65 \text { años reclutados } \\
\text { por cuatro médicos generalistas. }\end{array}$ & $\begin{array}{l}\text { Intervención ( } n=100) \text { : seis sesiones de consejo } \\
\text { sobre cesación tabáquica durante seis meses. } \\
\text { Control }(n=100) \text { : atención habitual y promoción } \\
\text { de un registro diario del consumo de cigarrillo } \\
\text { en dos sesiones durante seis meses. } \\
\text { Sin reporte acerca del entrenamiento o la } \\
\text { supervisión de los profesionales. }\end{array}$ & $\begin{array}{l}\text { Sin diferencias significativas a los seis meses de } \\
\text { terminada la intervención. }\end{array}$ \\
\hline Richmond 1995 & $\begin{array}{l}378 \text { pacientes de } 18 \text { a } 70 \text { años con elevado } \\
\text { consumo semanal de alcohol (más de } 35 \\
\text { tragos hombres y más de } 21 \text { las mujeres) } \\
\text { reclutados por } 119 \text { médicos generalistas. }\end{array}$ & $\begin{array}{l}\text { Intervención } 1 \text { ( } \mathrm{n}=96) \text { : programa de cambio de } \\
\text { conducta "Rastreo de alcohol", cinco sesiones } \\
\text { de cinco a } 25 \text { minutos durante cinco meses. } \\
\text { Intervención } 2 \text { ( } \mathrm{n}=96) \text { : consejo breve durante } \\
\text { una sesión de cinco minutos. } \\
\text { Control } 1(\mathrm{n}=93) \text { : estimación del } \\
\text { comportamiento de consumo y seguimiento. } \\
\text { Control } 2 \text { ( } \mathrm{n}=93 \text { ): solo cuidados habituales. } \\
\text { Todos los profesionales recibieron } \\
\text { entrenamiento. No se reporta si hubo } \\
\text { supervisión. }\end{array}$ & $\begin{array}{l}\text { Sólo se observó una tendencia a un menor } \\
\text { consumo en los asignados a la intervención } \\
\text { intensiva (grupo 1) especialmente en los } \\
\text { pacientes que asistieron a un mínimo de dos } \\
\text { sesiones. } \\
\text { A los } 12 \text { meses de terminada la intervención no } \\
\text { se observaron diferencias significativas en el } \\
\text { consumo de alcohol en los diferentes grupos ni en } \\
\text { la incidencia de problemas asociados al mismo. }\end{array}$ \\
\hline $\begin{array}{l}\text { Segnan } \\
1991\end{array}$ & $\begin{array}{l}923 \text { pacientes de } 20 \text { a } 60 \text { años tabaquistas } \\
\text { vírgenes de tratamiento, reclutados y } \\
\text { aleatorizados por } 44 \text { médicos generalistas. }\end{array}$ & $\begin{array}{l}\text { Intervención } 1 \text { ( } n=275) \text { : consejo reiterado, } 5 \\
\text { sesiones en } 9 \text { meses. . } \\
\text { Intervención } 2 \text { ( } n=294) \text { : consejo reiterado y } \\
\text { chicles de nicotina. } \\
\text { Intervención } 3(n=292) \text { : consejo reiterado y } \\
\text { realización de espirometría, } 1 \text { sesión. } \\
\text { Todos los profesionales fueron entrenados; sin } \\
\text { referencia a la supervisión.. }\end{array}$ & $\begin{array}{l}\text { Después de } 12 \text { meses de seguimiento: sin } \\
\text { diferencias significativas entre los grupos. }\end{array}$ \\
\hline
\end{tabular}




\section{Relato de una experiencia local}

Como podemos observar en la tabla 1, hay evidencia -por lo menos hasta la fecha relevada por Huibers y col. para la realización de su revisión sistemática publicada en 2008- de que el abordaje de resolución de problemas administrado por médicos generales es factible y en algunos casos efectivo para el tratamiento de pacientes con depresión mayor; y que es menos concluyente o limitada para el resto de las problemáticas estudiadas (trastornos por somatización, fatiga idiopática, abandono del tabaco o reducción del consumo de alcohol) más aún si se intenta comparar la efectividad de diferentes intervenciones entre sí ${ }^{5}$

Vale destacar que desde la creación en el Hospital Italiano de Buenos Aires de la Unidad de Medicina Familiar y Preventiva en 1989 y, posteriormente, del Servicio de Medicina Familiar y

\section{Cuadro 1: guía explicativa para la realización de la ficha del dispositivo FOCO.}

Comunitaria (SMFyC-HIBA) en 2008, los médicos de familia (MF) venimos trabajando en el marco de dicho paradigma, con la opción de tener espacios de supervisión directa e indirecta de nuestra práctica en sus aspectos psicosociales***.

Esta forma de trabajo tiene dos aspectos fundamentales:

1) Una modalidad de atención denominada FOCO (familia, orientación y contexto) a través de sucesivas entrevistas programadas $^{t+t}$ de cuarenta minutos de duración para la que el paciente debe solicitar con antelación dos turnos de atención con su médico de familia ${ }^{\ddagger \ddagger \ddagger}$ (cada turno contempla una entrevista de 20 minutos).

2) El uso de una ficha estructurada de registro ${ }^{\$ s}$ (ver cuadro 1 y su guía explicativa) que ayuda al profesional a que describa el motivo de la consulta, el contexto en el que está ocurriendo, su redefinición del problema o diagnóstico contextual operativo, la motivación del paciente y/o la familia para trabajar en dicho dispositivo, los objetivos del equipo terapéutico y el plan para la entrevista siguiente.

\section{Queja explícita del paciente}

Lo que el paciente percibe que le está sucediendo y trae a la consulta, usando sus mismas palabras. Puede estar expresado en el plano cognitivo, somático, emocional y/o de conducta.

\section{2. ¿Por qué ahora?}

Debe rastrearse específicamente qué hecho o secuencia de hechos tuvieron lugar para que la consulta surgiese en ese momento.

\section{Relación con el contexto}

Es importante conocer características del síntoma (dónde aparece, cuándo, cómo y cómo reaccionan las distintas personas involucradas). También es importante indagar factores atenuantes y perpetuadores del síntoma, creencias, temores y fantasías acerca del mismo, y de esta manera ayuda a discernir sobre distintos grados de estancamiento y afectación. ¿Qué interpreta el paciente que sucede? ¿Qué deja de hacer o quisiera hacer y no hace como consecuencia del problema?

\section{Redefinición del problema}

El MF considerando la información de 1 y 2 va construyendo con el paciente y/o familia un diagnóstico contextual operativo (DCO). Suele ocurrir que en dicho armado, el problema de consulta (astenia) se transforma en otro (depresión). A esto llamamos redefinición del problema, en el que el paciente llega con una queja o problema, y al ser evaluado por el médico y en esta construcción del DCO, se arriba a otro diagnóstico. Es importante que se trate de un problema "trabajable" (no son trabajables, por ejemplo, problemas económicos, o judiciales, pero sí sus consecuencias sobre la estructura familiar), con una meta alcanzable en un plazo de tiempo y observable en términos de conducta. También debe coincidir y ser coherente con lo que busca el paciente.

Algunos aspectos del problema a evaluar que nos proveen datos importantes para permitir el armado del DCO, son la relación con el contexto del paciente y las soluciones intentadas. De estas últimas es importante conocer si funcionaron o no, total o parcialmente. Es bueno reforzar aquellos intentos que funcionan o funcionaron alguna vez.

Las soluciones intentadas que se hicieron y fracasaron aportan al mantenimiento del problema, dan una pauta rápida para saber por dónde no hay que ir. La intensidad con que intentaron manejar el problema nos da una medida del grado de afectación y de la importancia con que se considera al problema.

\section{Motivación}

a) Referido al paciente: Qué busca al consultar y que esperaría de las entrevistas con el médico de familia. Qué está dispuesto a hacer. Qué expectativa tiene de esta serie de consultas.

b) Referido al MF: Qué grado de influencia piensa que tiene sobre este paciente o esta familia. Esto quiere decir que la motivación se haga viable en la concreción de un objetivo con una táctica y una estrategia.

\section{Objetivos}

Elegir conjuntamente un aspecto del motivo de consulta a trabajar.

Si es posible negociar una meta mínima a lograr a través de las entrevistas. Ej. ¿Cómo se daría/n cuenta que está/n mejorando? ¿Cuál sería la mínima pauta/cambio que le/s haría saber que está/n yendo en la dirección correcta?

\section{Plan para la próxima entrevista}

Maniobras o modalidades de acción que el MF considera convenientes para el logro de su objetivo. Qué hacer, qué evitar hacer, a quiénes citar

\section{Notas}

${ }^{\S}$ El Hospital Italiano de Buenos Aires es un Hospital Universitario Privado de Comunidad situado en la Ciudad de Buenos Aires en el que funciona un Seguro Privado de Salud que atiende población predominantemente de clase media y que vive en áreas urbanas y suburbanas de dicha ciudad. Este seguro se basa en un modelo semi-gerenciado de atención en el que cada paciente tiene un médico de cabecera (especialista en medicina familiar o en clínica médica) que se encarga de sus cuidados longitudinales.

${ }_{\star \star \star}$ Estos dispositivos de supervisión incluyen la atención del paciente o familia junto con un profesional especializado en salud mental, la grabación de entrevistas en video, la discusión de las mismas en ateneos de salud mental, espacios institucionalizados de supervisión individual del médico de familia respecto de su abordaje (y de la repercusión que sobre él tienen) hacia los pacientes con problemas psicosociales que él atiende.

t+tLas entrevistas deben ser programadas, si bien el sistema contempla la realización de entrevistas no programadas para el abordaje de problemas imprevistos que surgen en dicha entrevista (p.ej. duelo), que deben ser sucedidas de entrevistas programadas de cuarenta minutos de duración (doble turno).

\# Por el momento, esta modalidad de prestación sólo es válida para ser realizada por el médico de familia del paciente/familia en cuestión.

${ }^{85}$ Procuramos que este dispositivo tenga un foco de trabajo claro y definido, y que su duración sea limitada en el tiempo. Como vemos en el cuadro 2 , la ficha inicial consta de ocho ítems, existiendo también una ficha de seguimiento sin estructura definida que se utiliza para la/s entrevista/s sucesiva/s. 
En las entrevistas del dispositivo FOCO, los médicos de familia realizamos intervenciones individuales, de pareja $y / o$ de familia con el objetivo de movilizar los recursos del paciente y/o su grupo familiar, contribuir al desarrollo de estrategias para aliviar su padecer y/o para optimizar el desempeño de su sistema de cuidados.

Son candidatos al dispositivo FOCO aquellos pacientes/familias afectados por problemas psicosociales que puedan beneficiarse del mismo**** (ansiedad, depresión, crisis vitales, disfunción familiar, duelo, estrés del cuidador, etc.) así como por enfermedades crónicas, con problemas de adherencia al tratamiento, o para cambiar estilos de vida. De este modo, intentamos ayudarlos en sus procesos de cambio en relación con los problemas citados, con el fin último de que este sistema (el paciente y su familia) logren la mayor autonomía posible en su cuidado (autocuidado).

Cabe destacar que, más allá de los espacios semanales que brindan las Unidades Docentes Asistenciales ${ }^{t+t t}$ y de las instancias de supervisión individual con especialistas en salud mental del SMFyC-HIBA ${ }^{\ddagger \neq \neq \neq}$, tres veces por mes toman lugar sendos ateneos para que los diferentes profesionales del Servicio com- partan experiencias y reciban supervisión respecto de su abordaje de los problemas psicosociales de sus pacientes, así como de lo que les pasa a ellos mismos en relación a sus procesos de atención.

\section{Conclusiones generales}

En los últimos años cada vez más especialistas en medicina familiar realizamos este tipo de entrevistas psicosociales para el abordaje contextual de los problemas de nuestros pacientes y familias. Por ejemplo, durante 2009, 22 médicos del SMFyCHIBA realizamos 382 entrevistas enmarcadas en el dispositivo FOCO; durante 2010, 36 médicos realizamos 861 entrevistas; y durante 2011, 996 entrevistas por parte de 42 médicos.

Actualmente estamos desarrollando una investigación tendiente a describir los principales problemas que están siendo abordados en este dispositivo, así como la duración promedio de cada abordaje, la proporción de tratamientos que se consideraron concluidos y la calidad del registro por parte de los profesionales.

Recibido el 05/06/12 y aceptado el 10/09/12.

\section{Referencias}

1. Epstein R y col. Somatization reconsidered. Arch Intern Med 1999;159:215-222.

2. Allen $\mathrm{J}$ y col. The European definitions of the Key Features of the Discipline of General Practice, the role of the General Practitioner and a description of the Core Competencies of the General Practitioner / Family Physician. Prepared for WONCA EUROPE (The European Society of General Practice/ Family Medicine), 2002.

3. McWhinney I. 1994. Medicina de Familia. Madrid, Spain Mosby.

4. Huibers M y col. Intervenciones psicosociales por parte de médicos generales (Revisión Cochrane traducida). En: La Biblioteca Cochrane Plus, 2008 Número 2. Oxford: Update Software Ltd.

5. Engel G. The need for a new medical model: A challange for biomedicine. Science 1977,196:129-136.

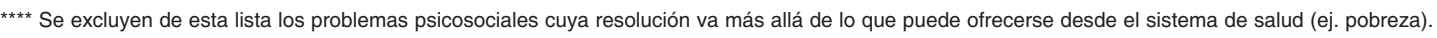
tttt Espacio de discusión que se reúne semanalmente y está integrado por profesionales de distinta experiencia: médicos de planta, médicos asociados, residentes.

\#\#\# Licenciados en Psicología o Terapeutas familiares.
} 